Gefässchirurgie 2011 · 16:229-229

DOI 10.1007/s00772-011-0917-z

๑) Springer-Verlag 2011

\author{
T. Noppeney \\ Versorgungszentrum für Gefäßmedizin, Nürnberg
}

\title{
Die Bedeutung der Varikose in der Gefäßchirurgie
}

hat in seinem Beitrag für die Gefäßchirurgie im Jahre 2008 auf die Bedeutung der venösen Erkrankungen im Gebiet der Gefäßchirurgie hingewiesen und mit Nachdruck gefordert, dass sie nicht vernachlässigt werden dürfen [1].

\section{$>$ Trotz hoher sozialer medizinischer Bedeutung fristet die Therapie von venösen Gefäßerkrankungen in vielen gefäßchirurgischen Einheiten ein Schattendasein}

Insbesondere unter dem Eindruck, dass sich die Möglichkeiten der Therapie der Varikose in den letzten 10 Jahren vervielfältigt haben, müssen wir als Gefäßchirurgen alles daran setzen, bei den technischen Entwicklungen mithalten zu können. So sollte sich jeder traditionsbewusste Varizenchirurg auch mit den modernen endovenösen Verfahren befassen, damit er zumindest deren Wertigkeit einschätzen kann. Das vorliegende Heft der Gefässchirurgie soll Ihnen einen Überblick über die verschiedenen Behandlungsoptionen bei dem Krankheitsbild der Varikose geben und uns allen vor Augen führen, dass wir bei der Datenlage der klassischen Varizenchirurgie im Vergleich zu den endovenösen Verfahren ein Problem mit der Evidenz haben.

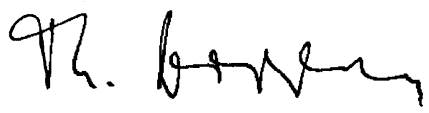

Dr. T. Noppeney

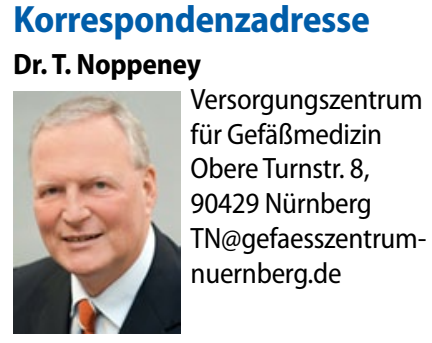

Interessenkonflikt. Der korrespondierende Autor weist auf folgende Beziehung hin: Beratertätigkeit für die Firma Covidien.

\section{Literatur}

1. Eklöf B (2008) Importance of venous diseas in the field of vascular surgery. Gefässchirurgie 13:429436

2. Nüllen H, Noppeney T (2010) Sozialmedizische und ökonomische Aspekte der Varikose. In: Noppeney T, Nüllen $\mathrm{H}$ (Hrsg) Lehrbuch Varikose, Diagnostik Therapie Begutachtung. Springer Berlin Heidelberg New York Tokio S. 62-65 